\section{Partielle oder radikale Nephrektomie?}

- Auf reges Interesse stießen die aktuellen Ergebnisse der Studie 30904 der European Organisation for Research and Treatment of Cancer (EORTC). In dieser Untersuchung nahmen insgesamt 541 Nierenkrebs-Patienten teil, die je zur Hälfte radikal oder partiell nephrektomiert wurden. Laut Prof. Dr. Hein van Poppel, Leuven/Belgien hat die Studie ergeben, dass eine nierenerhaltende chirurgische Behandlung einer radikalen Nephrektomie nicht unterlegen ist. Während der Nachbeobachtungszeit von rund neun Jahren hatten neun radikal nephrektomierte Patienten und zwölf nie- renerhaltend behandelte einen Progress. Zwölf Patienten verstarben an den Folgen des Nierenzellkarzinoms (vier im radikalen Arm, acht im konservativen) und drei an den Folgen der Operation (1 vs. 2). Die Gesamtüberlebenszeit war nach nierenerhaltender partieller $\mathrm{Ne}$ phrektomie signifikant kürzer $(\mathrm{p}=$ 0,032), die Komplikationsrate war leicht erhöht. Die Ergebnisse belegen nach Auffassung des Experten, dass die Chirurgie bei fortgeschrittenem Nierenzellkarzinom ein wesentlicher Therapiebaustein ist, wobei beide etablierten chirurgischen Verfahren ebenbürtig sind.

aam

\title{
Harnblasenrekonstruktion nach radikaler Zystektomie
}

— Eine radikale Zystektomie in Kombination mit einer pelvinen Lymphknotenentfernung ist die Standardtherapie bei muskelinvasivem Harnblasenkrebs. Durch eine nervenschonende Zystektomie können der Verlust der erektilen Funktion verhindert und eine Harnableitung ohne Stoma ermöglicht werden. Die Anlage einer intestinalen orthotopen Ersatzblase sei generell bei jedem zweiten zystektomierten Patienten machbar, so Prof. Dr. Urs E. Studer, Bern. Daher sollten sich alle Urologen, die radikale Zystektomien durchführen, mit nervenschonenden Operationsverfahren auseinandersetzen. Außerdem sollten sie Kenntnisse erwerben, wie eine orthotope intestinale Blasenrekonstruktion mit guten funktionellen Ergebnissen durchgeführt wird. Als wichtigste Voraussetzungen dafür nannte Studer eine sorgfältige Patientenselektion, Kenntnis der entscheidenden Handgriffe peri- und postoperativ sowie ein proaktives Management der Patienten. aam

\section{Operationsroboter versus Laparoskopie}

— Laparoskopische Eingriffe sind bei vielen urologischen Operationen Standard. Sie sind jedoch mit einigen Nachteilen verbunden, wie etwa einer flachen Lernkurve und schwierig durchzuführende rekonstruktive Techniken. Die Roboter-assistierte Laparoskopie bietet den Vorteil einer minimal invasiven Chirurgie sowie eines genaueren und flexibleren Operierens, während gleichzeitig die Lernkurve des Operateurs und seine Fähigkeit zur Durchführung schwieriger Operationen verbessert werden. RoboterAssistenz wird derzeit hauptsächlich bei Prostatakrebs genutzt. Die onkologischen und funktionellen Ergebnisse sind laut Dr. Alex Mottrie, Aalst/Belgien, vielversprechend, was allerdings noch durch Langzeitergebnisse bestätigt werden müsse. Dank der Vorteile der robotischen Manipulationen können nun auch schwierigere Operationen erfolgreich minimal invasiv durchgeführt werden, beispielsweise Pyeloplasik, partielle Nephrektomie, Zystektomie und die laparoskopische Konstruktion der Harnableitung. Mit zunehmender Erfahrung in der Robotor-unterstützten Chirurgie können auch andere Indikationen, ablative wie rekonstruktive, in Angriff genommen werden.

\section{Interventionelle Therapieverfahren bei Prostatahyperplasie}

- In den letzten 15 Jahren wurden eine Vielzahl neuer chirurgischer Techniken für die Behandlung von Blasenauslassobstruktionen im Rahmen einer Prostatahyperplasie (BPH) vorgestellt. Referenz für alle neu entwickelten operativen $\mathrm{BPH}-$ Therapien sei nach wie vor die transurethrale Prostataresektion (TURP) des hyperplastischen Gewebes, so Dr. Ken Anson, London. Eine praktikable Alternative zur TURP sieht der Experte in der Kalium-Titanyl-Phosphat (KTP)Laservaporisation. Die Technik sei zwar aufwendig, aber ermögliche nach seiner bisherigen Erfahrung erstmals vergleich- bar gute klinische Resultate wie die TURP. Wesentliche Vorteile seien das geringere Blutungsrisiko und die kürzere Krankenhausverweildauer. Auch Patienten, die für eine TURP nicht geeignet erscheinen, wie etwa antikoagulierte Patienten, sind Kandidaten für den KTPLaser. Doch eines dürfe man nicht vergessen, betonte Anson. Parallel zu der Entwicklung der Lasertechniken habe die TURP eine "Revolution “ durchgemacht mit verbesserten Endoskopen und Diathermie-Schleifen. Als Alternative zur monopolaren TURP wurden bipolare Technologien entwickelt, die als Spüllö- sung physiologische Kochsalzlösungen verwenden. Gegenüber der monopolaren TURP hat die bipolare TURP den Vorteil einer kürzeren Dauerkatheterliegezeit und einer kürzeren Hospitalisierung. Letztendlich können erst langfristig angelegte randomisierte Studien Auskunft darüber geben, welches der verschiedenen Verfahren in Bezug auf Langzeitergebnisse, Sicherheit und Kosteneffizienz überlegen ist.

aam 